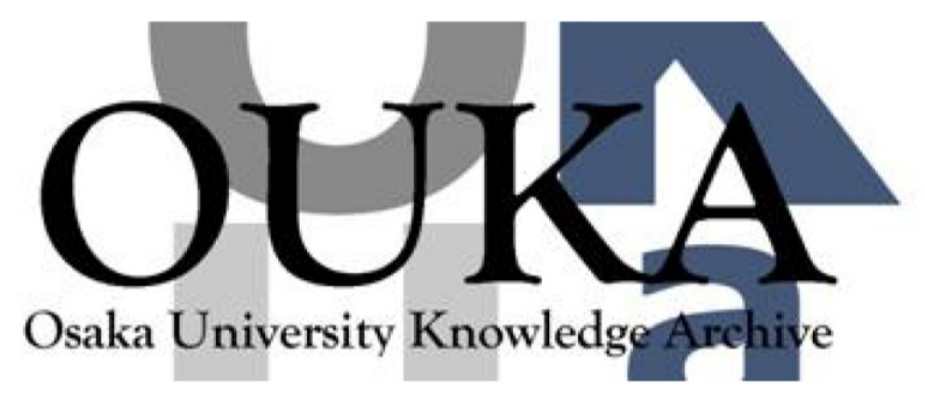

\begin{tabular}{|c|l|}
\hline Title & $\begin{array}{l}\text { A } 0.5 \text { V Area-Efficient Transformer Folded- } \\
\text { Cascode CMOS Low-Noise Amplifier }\end{array}$ \\
\hline Author(s) & $\begin{array}{l}\text { Kihara, Takao; Park, Hae-Ju; Takobe, Isao et } \\
\text { al. }\end{array}$ \\
\hline Citation & $\begin{array}{l}\text { IEICE Transactions on Electronics. E92-C(4) } \\
\text { p.564-p. } 575\end{array}$ \\
\hline Issue Date & $2009-04$ \\
\hline oaire:version VoR \\
\hline URL & https://hdl. handle. net/11094/51701 \\
\hline rights & copyright $\odot 2009$ IEICE \\
\hline Note & \\
\hline
\end{tabular}

Osaka University Knowledge Archive : OUKA

https://ir. Library. osaka-u. ac. jp/

Osaka University 


\title{
A 0.5 V Area-Efficient Transformer Folded-Cascode CMOS Low-Noise Amplifier
}

\author{
Takao KIHARA ${ }^{\dagger a)}$, Hae-Ju PARK ${ }^{\dagger}$, Isao TAKOBE ${ }^{\dagger}$, Fumiaki YAMASHITA ${ }^{\dagger}$, Nonmembers \\ Toshimasa MATSUOKA ${ }^{\dagger}$, and Kenji TANIGUCHI ${ }^{\dagger}$, Members
}

\begin{abstract}
SUMMARY A $0.5 \mathrm{~V}$ transformer folded-cascode CMOS low-noise amplifier (LNA) is presented. The chip area of the LNA was reduced by coupling the internal inductor with the load inductor, and the effects of the magnetic coupling between these inductors were analyzed. The magnetic coupling reduces the resonance frequency of the input matching network, the peak frequency and magnitude of the gain, and the noise contributions from the common-gate stage to the LNA. A partially-coupled transformer with low magnetic coupling has a small effect on the LNA performance. The LNA with this transformer, fabricated in a $90 \mathrm{~nm}$ digital CMOS process, achieved an $S_{11}$ of $-14 \mathrm{~dB}, \mathrm{NF}$ of $3.9 \mathrm{~dB}$, and voltage gain of $16.8 \mathrm{~dB}$ at $4.7 \mathrm{GHz}$ with a power consumption of $1.0 \mathrm{~mW}$ at a $0.5 \mathrm{~V}$ supply. The chip area of the proposed LNA was $25 \%$ smaller than that of the conventional folded-cascode LNA.
\end{abstract}

key words: CMOS, low-noise amplifier (LNA), low voltage, transformer

\section{Introduction}

Although the continuous scaling of CMOS technologies has improved the high-frequency performance of MOSFETs, it has imposed two challenges on CMOS radio-frequency integrated circuits (RFICs): low-voltage operation and a small chip area. The International Technology Roadmap for Semiconductors (ITRS) [1] predicts that the supply voltages of low-power digital circuits will decrease to $0.5 \mathrm{~V}$ in the near future. Reference [2] shows that a $45 \mathrm{~nm}$ (stateof-the-art) CMOS process costs approximately 10 times as much as a $0.13 \mu \mathrm{m}$ (most widely used) CMOS process. Considering the integration of RF circuits with digital circuits (i.e., system-on-a-chip), we need to develop low-voltage and small-area (low-cost) RF circuits.

Low-noise amplifiers (LNAs) have difficulty in operating at low voltages with a small chip area. Cascode LNAs with inductive source degeneration [3] have been widely used for narrow band receivers, due to low-noise performance and good input impedance matching. However, this LNA is not suitable for low-voltage operation, because it requires a supply voltage of more than two drain-source saturation voltages $\left(V_{D D}>2 V_{D S, \text { sat }}\right)$ to operate the cascode transistor. Although folded-cascode LNAs [4], [5] are more suitable for low voltage operation $\left(V_{D D}>V_{D S \text {, sat }}\right)$, they require more inductors, which lead to an increase in the chip area. Other reported low-voltage LNAs [6], [7] consume

\footnotetext{
Manuscript received July 31, 2008.

Manuscript revised October 27, 2008.

${ }^{\dagger}$ The authors are with the Division of Electrical, Electronics and Information Engineering, Graduate School of Engineering, Osaka University, Suita-shi, 565-0871 Japan.

a)E-mail: kihara@si.eei.eng.osaka-u.ac.jp DOI: 10.1587/transele.E92.C.564
}

much larger chip area than the folded-cascode LNAs, due to many inductors.

We propose a $0.5 \mathrm{~V}, 5 \mathrm{GHz}$ transformer folded-cascode CMOS LNA [8], which has a smaller chip area than the conventional folded-cascode LNA. The transformer that consists of the internal and load inductors reduces the chip area of the LNA, while affecting the LNA performance. This paper is organized as follows. Section 2 describes the circuit topology of the proposed LNA. The effects of the transformer on the LNA performance are analyzed in Sect. 3. Section 4 describes the design of the LNA and transformer. Section 5 presents the measurements of the LNA implemented in a $90 \mathrm{~nm}$ digital CMOS technology, and Sect. 6 concludes the paper.

\section{Circuit Topology}

Figure 1 shows a schematic of the proposed LNA based on the conventional folded-cascode LNA with inductive source degeneration. The gate and source inductors, $L_{g}$ and $L_{s}$, provide input impedance matching at an operating frequency [3]. The PMOS transistor $M_{2}$ reduces the Miller effect of the gate-drain capacitor of the input transistor $M_{1}$, and improves the reverse isolation performance of the LNA. The internal inductor $L_{I}$, resonating with the parasitic capacitance $C_{I}$ at node I, provides a high impedance, thereby the signal current amplified by $M_{1}$ flows into $M_{2}$. The load inductor $L_{L}$ also resonates with the parasitic capacitance $C_{L}$, resulting in a high impedance. These inductors, $L_{I}$ and $L_{L}$, are magnetically coupled to form a transformer in such a way as to have a positive magnetic coupling with retaining the LNA

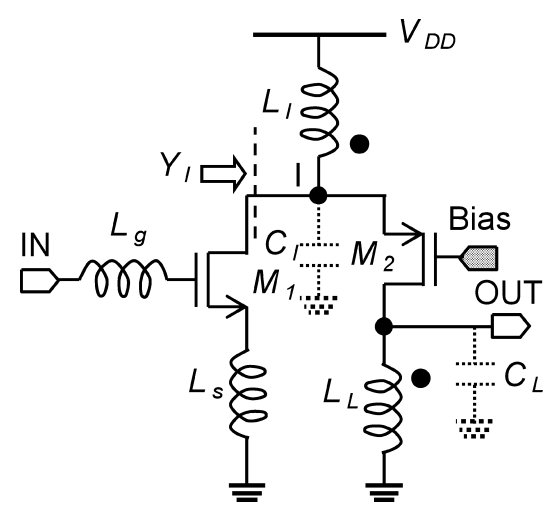

Fig. 1 Schematic of the proposed LNA. 
performance.

The positive magnetic coupling of $L_{I}$ and $L_{L}$ is the most effective way to reduce the chip area of the folded-cascode LNA. Increasing the magnetic coupling leads to a smaller $L_{I}$ and $L_{L}$ (smaller chip area), as will be shown in the next section. On the contrary, the negative magnetic coupling requires a larger $L_{I}$ and $L_{L}$ (larger chip area). The coupling of $L_{g}$ or $L_{s}$ and $L_{I}$ or $L_{L}$ is also not beneficial for the following reasons:

1. $L_{g}$ is often implemented with a bonding wire.

2. $L_{s}$ is usually small $(<1.0 \mathrm{nH})$ for input impedance matching.

3. The coupling makes the LNA unstable.

\section{Effect of Magnetic Coupling}

The magnetic coupling between $L_{I}$ and $L_{L}$ affects the LNA performance in terms of input impedance, gain, and noise. In this section, the effects of the magnetic coupling are analyzed, and the stability of the LNA is also discussed.

\subsection{Input Impedance}

The magnetic coupling changes the frequency response of the LNA input impedance $Z_{\text {in }}$ through the gate-drain capacitance of $M_{1}, C_{g d 1}$. The small-signal equivalent circuit of the input stage, shown in Fig. 2, yields $Z_{\text {in }}$, given by

$$
\begin{aligned}
& Z_{\text {in }} \approx s L_{g}+\frac{\omega_{T 1} L_{s}+s L_{s}+\frac{1}{s C_{g s 1}}}{\alpha_{M}}, \\
& \alpha_{M}=1+\alpha_{g d 1}+\frac{\alpha_{g d 1} g_{m 1}}{Y_{I}},
\end{aligned}
$$

where $\omega_{T_{1}}=g_{m 1} / C_{g s 1}$ is the unity current gain frequency of $M_{1} ; \alpha_{g d 1}=C_{g d 1} / C_{g s 1}$ is the ratio between $C_{g d 1}$ and $C_{g s 1}$. The input admittance of the common-gate stage, shown in Fig. 3, is given by

$$
\begin{aligned}
Y_{I}=\frac{i_{i}}{v_{i}} \approx & g_{m 2}+s C_{I}+\frac{1}{s L_{I}+R_{I}} \\
& -\frac{n k\left(g_{m 2}-s n k C_{L}\right)}{1+s^{2}\left(1-k^{2}\right) L_{L} C_{L}+s R_{L} C_{L}},
\end{aligned}
$$

where $g_{m 2}$ is the transconductance of $M_{2} ; R_{I}$ and $R_{L}$ are the parasitic resistances of $L_{I}$ and $L_{L}$, respectively; $k$ and $n=\sqrt{L_{L} / L_{I}}$ are the coupling factor and turns ratio of the transformer, respectively. The frequency responses of $Y_{I}$ and $1 / Y_{I}$ are described in Appendix A. The calculated real and imaginary parts of $\alpha_{M}$ and $Z_{i n}$ are shown in Figs. 4(a) and (b), respectively. Using $\operatorname{Re}\left[\alpha_{M}\right]$ and $\operatorname{Im}\left[\alpha_{M}\right]$, we can approximate $Z_{\text {in }}$ as

$$
\begin{aligned}
\operatorname{Re}\left[Z_{i n}\right] & \approx \frac{\omega_{T 1} L_{s} \cdot \operatorname{Re}\left[\alpha_{M}\right]-\frac{\operatorname{Im}\left[\alpha_{M}\right]}{\omega C_{g s 1}}}{\left|\alpha_{M}\right|^{2}}, \\
\operatorname{Im}\left[Z_{\text {in }}\right] & \approx \omega L_{g}-\frac{\omega_{T 1} L_{s} \cdot \operatorname{Im}\left[\alpha_{M}\right]+\frac{\operatorname{Re}\left[\alpha_{M}\right]}{\omega C_{g s 1}}}{\left|\alpha_{M}\right|^{2}},
\end{aligned}
$$

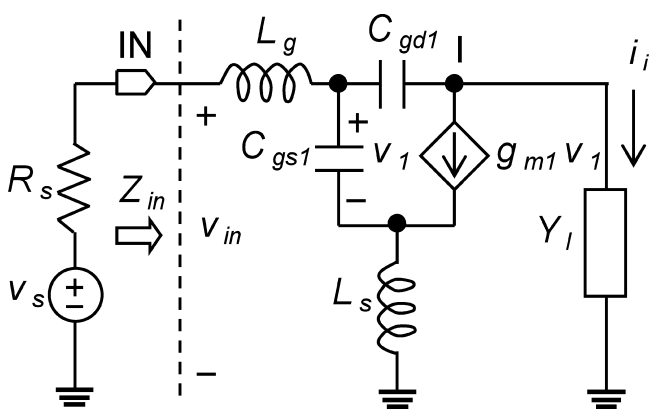

Fig. 2 Small-signal equivalent circuit of the input stage.

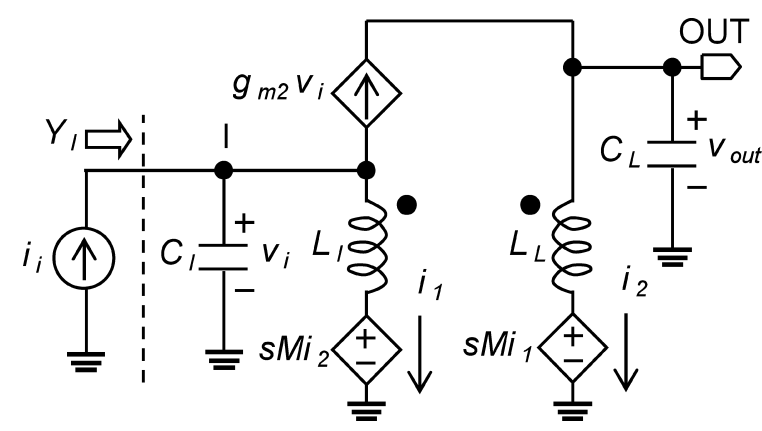

Fig. 3 Small-signal equivalent circuit of the common-gate stage

where $\omega L_{s}$ is ignored against $1 / \omega C_{g s}$. As shown in Fig. 4(a), $\operatorname{Re}\left[\alpha_{M}\right]$ increases and $\operatorname{Im}\left[\alpha_{M}\right]$ decreases at low frequencies, which results in an increase of $\operatorname{Re}\left[Z_{i n}\right]$. For a low $k(<0.6), \operatorname{Re}\left[Z_{i n}\right]$ becomes a maximum around the frequency at which $\operatorname{Im}\left[\alpha_{M}\right]$ becomes a minimum. Meanwhile, $\operatorname{Im}\left[Z_{\text {in }}\right]$ with $k$ reaches zero faster than $\operatorname{Im}\left[Z_{i n}\right]$ without $k(k=0)$, due to the increase in $\operatorname{Re}\left[\alpha_{M}\right]$ at low frequencies.

The magnetic coupling shifts the input impedance matching region (an $S_{11}$ of less than $-10 \mathrm{~dB}$ ) toward lower frequencies. Due to good reverse isolation of the foldedcascode topology $\left(S_{12} \simeq 0\right)$, the $S_{11}$ of the LNA can be approximated as [9]

$$
S_{11} \approx \frac{Z_{i n}-R_{s}}{Z_{\text {in }}+R_{s}},
$$

where $R_{s}$ is the signal source impedance. For input impedance matching at the frequency of $\omega_{0}$, the following conditions must be satisfied:

$$
\begin{aligned}
& \operatorname{Re}\left[Z_{\text {in }}\right] \simeq R_{s}, \\
& \operatorname{Im}\left[Z_{\text {in }}\right] \simeq 0, \\
& \operatorname{Im}\left[\alpha_{M}\right] \simeq 0,
\end{aligned}
$$

which give the following conditions:

$$
\begin{aligned}
& \frac{\omega_{T 1} L_{s}}{\operatorname{Re}\left[\alpha_{M}\left(j \omega_{0, \alpha_{M}}\right)\right]} \simeq R_{s}, \\
& \omega_{0} \approx \frac{1}{\sqrt{L_{g} C_{g s 1} \cdot \operatorname{Re}\left[\alpha_{M}\left(j \omega_{0, \alpha_{M}}\right)\right]}}, \\
& \omega_{0, \alpha_{M}} \approx \frac{1}{\sqrt{L_{I}\left(C_{I}+n^{2} k^{2} C_{L}\right)}},
\end{aligned}
$$



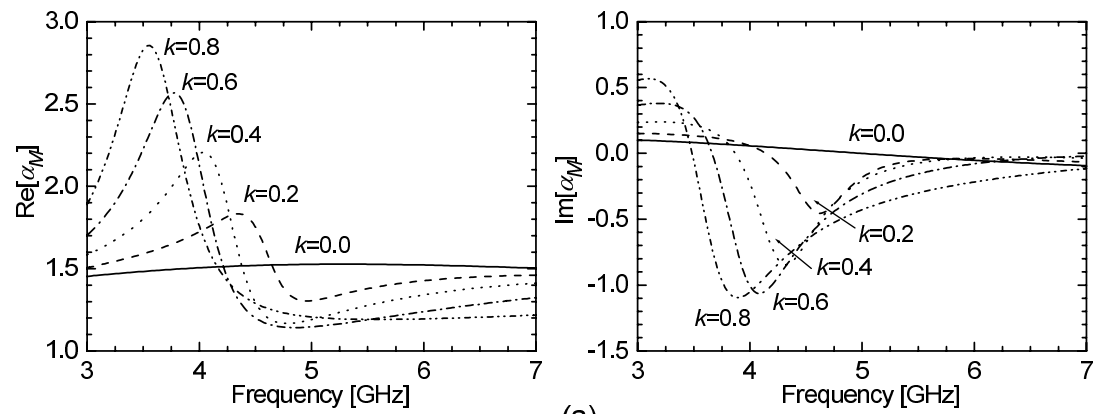

$g_{m 1}=20 \mathrm{mS}, L_{g}=4.0 \mathrm{nH}, L_{s}=0.6 \mathrm{nH}$, $C_{g s 1}=150 \mathrm{fF}, C_{g d 1}=35 \mathrm{fF}, g_{m 2}=15 \mathrm{mS}$,

(a)
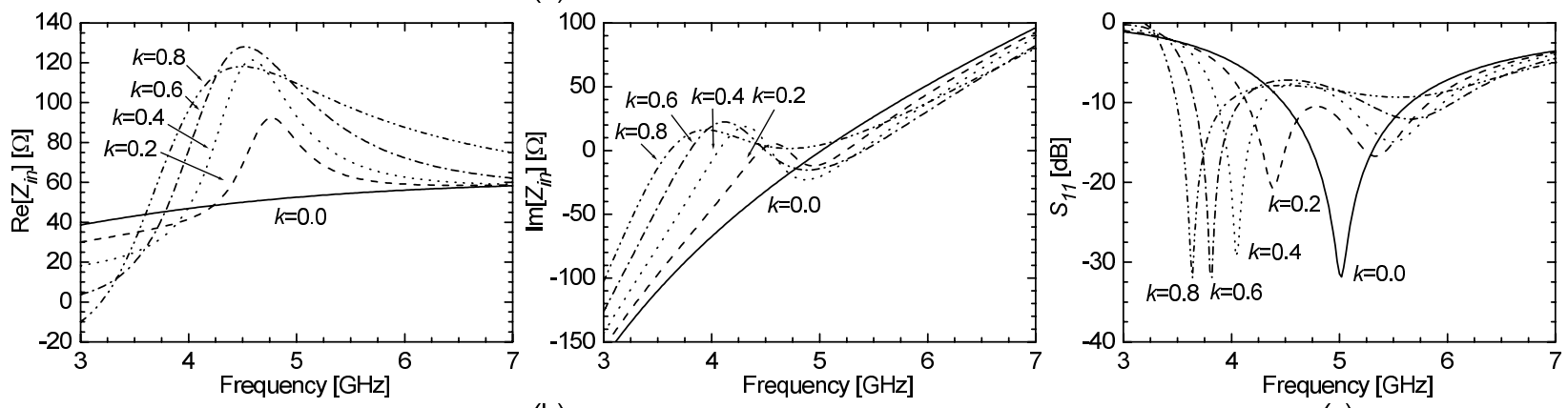

(b)

(c)

Fig. 4 Calculated (a) $\alpha_{M}$, (b) $Z_{i n}$, and (c) $S_{11}$ with $k$ as a parameter.

respectively, where $\operatorname{Re}\left[\alpha_{M}\left(j \omega_{0, \alpha_{M}}\right)\right]$ is approximated as

$$
\operatorname{Re}\left[\alpha_{M}\left(j \omega_{0, \alpha_{M}}\right)\right] \approx 1+\alpha_{g d 1}+\frac{\alpha_{g d 1} g_{m 1}}{(1-n k) g_{m 2}} .
$$

Equations (10)-(13) show that the resonance frequencies of $Z_{\text {in }}$ and $\alpha_{M}$ decrease and $\operatorname{Re}\left[\alpha_{M}\right]$ increases as $k$ increases. Figure 4(c) shows the calculated $S_{11}$ with $k$ as a parameter. Input impedance matching is achieved around $\omega_{0}$, which decreases with increasing $k$.

\subsection{Gain}

The magnetic coupling reduces the peak frequency and magnitude of the LNA gain. The common-gate stage acts as a transimpedance, which converts the input signal current $i_{i}$ to the output voltage $v_{\text {out }}$, as shown in Fig. 3. The input current $i_{i}$ amplified by the first stage is derived from Fig. 2:

$$
i_{i} \approx-\frac{g_{m 1}}{j \omega_{0} C_{g s 1} R_{s} \cdot \operatorname{Re}\left[\alpha_{M}\right]} v_{i n}
$$

where input impedance matching is assumed (i.e., $Z_{\text {in }}=R_{s}$ ) and $v_{\text {in }}$ represents the input voltage of the LNA as shown in Fig. 2. The transimpedance from node I to the output is given by

$$
\begin{aligned}
Z_{T} & =\frac{v_{\text {out }}}{i_{i}}=\frac{v_{\text {out }}}{v_{i}} \frac{v_{i}}{i_{i}} \\
& =\frac{n k+g_{m 2}\left[R_{L}+s\left(1-k^{2}\right) L_{L}\right]}{1+s C_{L}\left[R_{L}+s\left(1-k^{2}\right) L_{L}\right]} \cdot \frac{1}{Y_{I}} \\
& =\frac{\frac{n k}{R_{L}+s\left(1-k^{2}\right) L_{L}}+g_{m 2}}{D}, \\
D & =\left(s C_{I}+\frac{1}{s L_{I}+R_{I}}\right)\left(s C_{L}+\frac{1}{s\left(1-k^{2}\right) L_{L}+R_{L}}\right)
\end{aligned}
$$

$$
\begin{aligned}
& +g_{m 2}\left(s C_{L}+\frac{1-n k}{s\left(1-k^{2}\right) L_{L}+R_{L}}\right) \\
& +\frac{s n^{2} k^{2} C_{L}}{s\left(1-k^{2}\right) L_{L}+R_{L}} .
\end{aligned}
$$

Around $\omega=1 / \sqrt{L_{I} C_{I}}=1 / \sqrt{L_{L} C_{L}}$, the first term in Eq. (16) is approximated by zero:

$$
D \approx g_{m 2}\left(j \omega C_{L}+\frac{1-n k}{j \omega\left(1-k^{2}\right) L_{L}}\right)+\frac{n^{2} k^{2} C_{L}}{\left(1-k^{2}\right) L_{L}},
$$

where $R_{I}$ and $R_{L}$ are ignored for simplicity. The magnitude of $Z_{T}$ becomes a maximum when the first term in Eq. (17) equals zero. The voltage gain of the LNA and its peak frequency can be therefore expressed as

$$
\begin{aligned}
A_{v, L N A} & =\left|\frac{v_{\text {out }}}{v_{\text {in }}}\right| \\
& =\left|\frac{i_{i} Z_{T}}{v_{\text {in }}}\right|=\left|\frac{g_{m 1} Z_{T}}{j \omega_{0} C_{g s 1} R_{s} \cdot \operatorname{Re}\left[\alpha_{M}\right]}\right|, \\
\omega_{p} \approx & \frac{1}{\sqrt{\frac{1-k^{2}}{1-n k} L_{L} C_{L}}},
\end{aligned}
$$

respectively. Equations (18) and (19) show that the magnitude and peak frequency of the voltage gain decrease with increasing $k$. Figure 5 shows the calculated $A_{v, L N A}$ with $k$ as a parameter. The increase of $k$ shifts the gain peak toward a lower frequency and reduces the gain magnitude. The peak frequency in Fig. 5 corresponds well to that calculated from Eq. (19).

The LNA with the magnetic coupling sacrifices a maximum voltage gain to achieve the target peak frequency, $\omega_{p, t}$. 


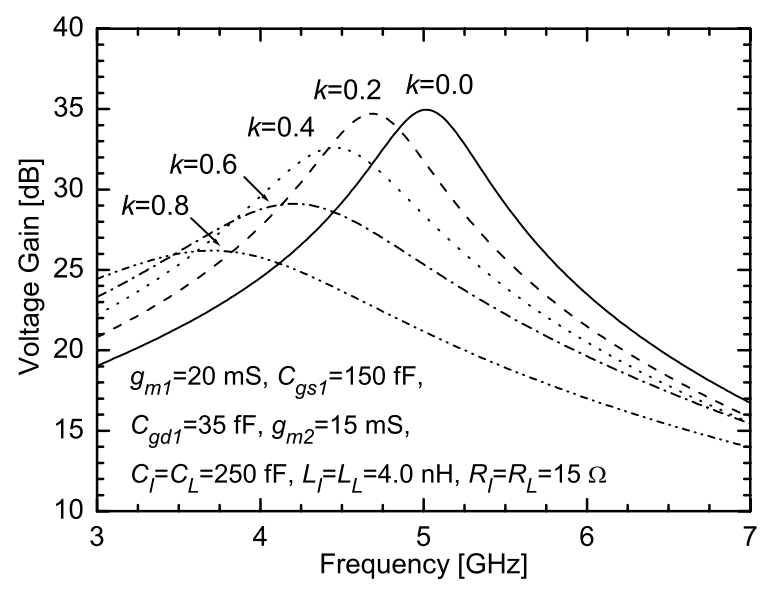

Fig. 5 Calculated voltage gain with $k$ as a parameter.

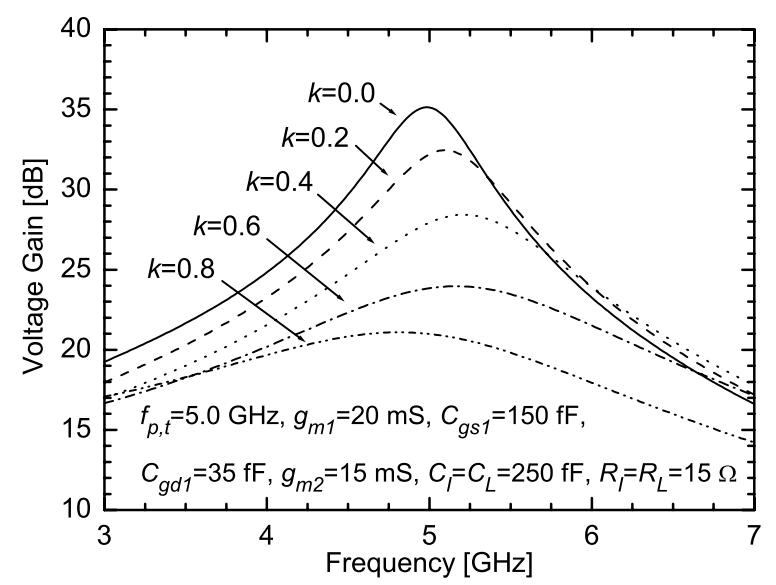

Fig. 6 Calculated voltage gain with $k$ as a parameter for $L_{L}=L_{I}=$ $1 / \omega_{p, t}^{2}(1+k) C_{L}$.

Equation (19) gives the following condition:

$$
L_{L}=L_{I}=\frac{1}{\omega_{p, t}^{2}(1+k) C_{L}} \quad(\text { for } n=1) .
$$

A turns ratio of one provides the smallest chip area of the transformer, because $L_{L}$ and $L_{I}$ simultaneously decrease with increasing $k$. Equation (20) shows that a smaller $L_{I}$ and $L_{L}$ are required to achieve $\omega_{p, t}$ as $k$ increases. Reducing $L_{I}$ and $L_{L}$ leads to a smaller chip area, but to a decrease in the parallel impedances of the internal and load LC tanks at the resonance frequencies $\left(Z_{p} \approx\left(\omega_{0} L_{I, L}\right)^{2} / R_{I, L}\right)$, causing a lower voltage gain. Figure 6 shows the calculated voltage gain for $f_{p, t}=5.0 \mathrm{GHz}$ where $L_{L}$ and $L_{I}$ satisfy Eq. (20). A peak frequency of approximately $5.0 \mathrm{GHz}$ can be achieved even for a large $k$, while the maximum gain decreases with increasing $k$. A small coupling factor such as 0.2 , however, is acceptable for the LNA, due to a small gain reduction of $3 \mathrm{~dB}$.

\subsection{Noise}

The transformer reduces the output noise originating from

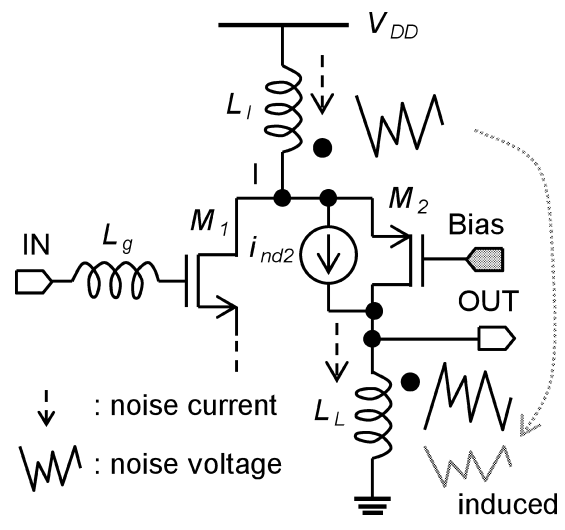

Fig. 7 Mechanisms for noise reduction of $i_{n d 2}$.

the common-gate transistor and the parasitic resistance of $L_{L}$, thereby improving the noise performance. This transformer noise reduction scheme has been reported in [10]. Figure 7 conceptually illustrates how the transformer reduces the drain noise current of $M_{2}$, represented by $i_{n d 2}$. The primary (internal) inductor $L_{I}$ detects $i_{n d 2}$, and then induces a noise voltage to the secondary (load) inductor $L_{L}$. The induced noise voltage is correlated and anti-phase to the output noise voltage produced by $i_{n d 2}$ flowing through $L_{L}$, reducing the output noise caused by $M_{2}$. The other output noise originating from $L_{L}$ is also reduced by the transformer in the same way.

The magnetic coupling affects the noise contributions from $M_{2}, L_{I}$, and $L_{L}$ to the LNA $\left(F_{M_{2}}, F_{L_{I}}\right.$, and $F_{L_{L}}$, respectively), but not that from $M_{1}\left(F_{M_{1}}\right)$. The LNA noise factor is given by

$$
\begin{aligned}
& F=1+F_{M_{1}}+F_{M_{2}}+F_{L_{I}}+F_{L_{L}}, \\
& F_{M_{1}} \approx \frac{\gamma_{1} \chi_{1}}{\alpha_{1}} g_{m 1} R_{s}\left(\frac{\omega_{0}}{\omega_{T_{1}}}\right)^{2}+\frac{\alpha_{1} \delta_{1}}{\kappa_{1} g_{m 1} R_{s}}, \\
& \chi_{1}=\left(1+\alpha_{g d 1}\right)^{2}-2|c| \alpha_{1} \sqrt{\frac{\delta_{1}}{\kappa_{1} \gamma_{1}}}\left(1+\alpha_{g d 1}\right)+\frac{\alpha_{1}^{2} \delta_{1}}{\kappa_{1} \gamma_{1}}, \\
& F_{M_{2}} \approx 4\left|\frac{Y_{I}}{Y_{0}+Y_{I}}\right|^{2} \frac{\gamma_{2}}{\alpha_{2}} g_{m 2} R_{S}\left(\frac{\omega_{0}}{\omega_{T_{1}}}\right)^{2}\left|\alpha_{M}\right|^{2} \\
& F_{L_{I}} \approx 4\left|\frac{Y_{I}}{Y_{0}+Y_{I}}\right|^{2} R_{I} R_{S}\left(\frac{\omega_{0}}{\omega_{T_{1}}}\right)^{2}\left|\alpha_{M}\right|^{2} \\
& \times\left|\frac{(1-k) g_{m 2}-k\left(Y_{0}+j \omega_{0} C_{I}\right)}{k+j \frac{g_{m 2}(1-k)}{\omega_{0} C_{L}}}\right|^{2}, \\
& F_{L_{L}} \approx 4\left|\frac{Y_{I} C_{L_{I}} C_{L}}{Y_{0}+Y_{I}}\right|^{2} R_{L} R_{s}\left(\frac{\omega_{0}}{\omega_{T_{1}}}\right)^{2}\left|\alpha_{M}\right|^{2} \\
& \times\left|\frac{(1-k) g_{m 2}+Y_{0}+Y_{L_{I} C_{I}}}{k+j}\right|^{2},
\end{aligned}
$$

where $\alpha_{i}=g_{m i} / g_{d 0 i}$ and $g_{d 0 i}$ is the zero bias drain conduc- 


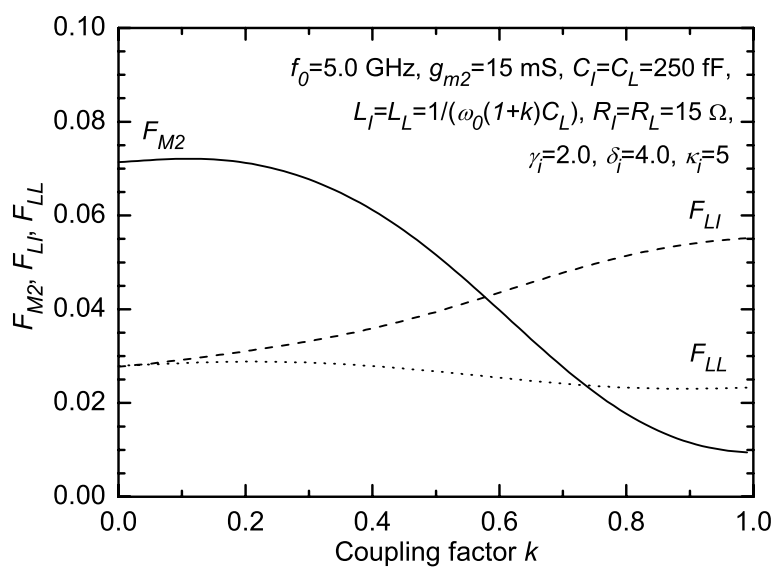

Fig. 8 Calculated $F_{M_{2}}, F_{R_{I}}$, and $F_{R_{L}}$ versus $k$.

tance of $M_{i}(i=1,2) ; \gamma_{i}$ and $\delta_{i}$ are the drain noise current factor and the induced gate noise current factor, respectively, and $c$ is the correlation coefficient between these noise currents $(\simeq j 0.395[11]) ; \kappa_{i}$ is the Elmore constant $(=5[12])$; $L_{I}=L_{L}=1 / \omega_{0}^{2}(1+k) C_{L} ; Y_{0}$ represents the output admittance of the input stage at node I and is approximated as $j \omega C_{g d 1} ; Y_{L_{I} C_{I}}=j \omega C_{I}+1 /\left(j \omega L_{I}+R_{I}\right)$. The detailed derivations are summarized in Appendix B. Equations (22)(26) show that $F_{M_{1}}$ is independent of $k$ while $F_{M_{2}}, F_{L_{I}}$, and $F_{L_{L}}$ are functions of $k$. Figure 8 shows the calculated $F_{M_{2}}$, $F_{L_{l}}$, and $F_{L_{L}}$ versus $k$. As Eq. (24) shows, $F_{M_{2}}$ approaches zero with increasing $k$. Meanwhile, $F_{L_{I}}$ increases and $F_{L_{L}}$ slightly decreases. This difference originates from the different numerators in Eqs. (25) and (26), i.e., $-k\left(Y_{0}+j \omega_{0} C_{I}\right)$ and $Y_{0}+Y_{L_{I} C_{I}}$.

The noise improvement by the transformer is limited in the folded-cascode topology. The calculated noise figure (NF, defined by $10 \log F$ ) versus $k$ are shown in Fig. 9, where $90 \mathrm{~nm}$ CMOS process parameters were used. The NF simulated using Agilent Advanced Design System (ADS) are also plotted. Figure 9 shows that the calculated NF is comparable to the simulated NF, and the magnetic coupling reduces the NF by up to $0.08 \mathrm{~dB}$ (calculated) or $0.12 \mathrm{~dB}$ (simulated) for $g_{m 2}=15 \mathrm{mS}$. The amount of noise reduction is relatively small, because the noise of $M_{1}$ is the dominant noise source in the LNA $\left(F_{M_{1}} \simeq 1.20\right.$ and 1.05 in the calculations and simulations, respectively).

\subsection{Stability}

A small $L_{I}$ or large $C_{L}$ ensures the stability of the LNA. The proposed LNA becomes potentially unstable, because the transformer provides a positive feedback from the output to node I, as shown in Fig. 1. For stability, the LNA must satisfy the following condition [9]:

$$
\operatorname{Re}\left[Z_{i n}\right]>0 .
$$

For $k=1$ and low frequencies (the worst case), $\operatorname{Re}\left[Z_{\text {in }}\right]$ is approximated as

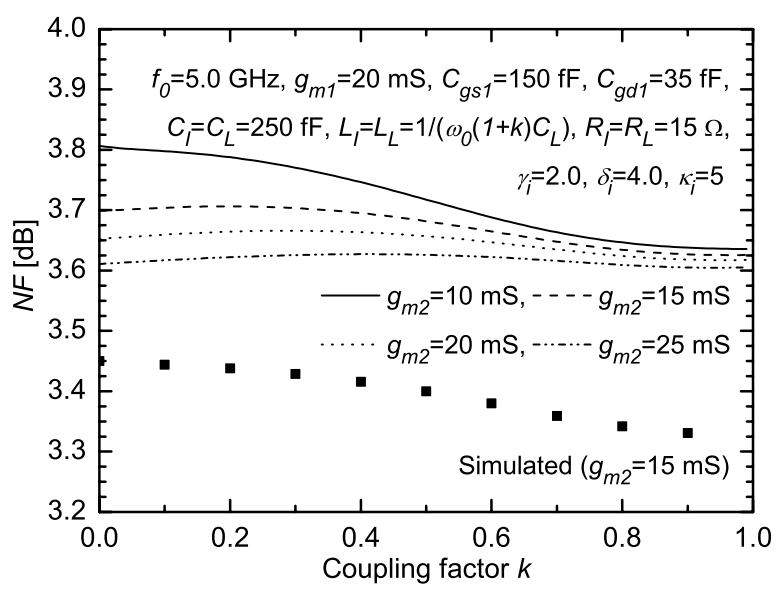

Fig. 9 Calculated and simulated NFs versus $k$.

$$
\operatorname{Re}\left[Z_{i n}\right] \approx \frac{\omega_{T 1}\left[L_{s}\left(1+\alpha_{g d 1}\right)-L_{I} \alpha_{g d 1}\right]}{\left(1+\alpha_{g d 1}\right)^{2}+\omega^{2} L_{I}^{2} \alpha_{g d 1}^{2} g_{m 1}^{2}} .
$$

Substituting Eq. (28) in Eq. (27), we have

$$
L_{I}<L_{s}\left(1+\frac{1}{\alpha_{g d 1}}\right),
$$

which shows that a smaller $L_{I}$ ensures the stability. Using Eq. (20), we can rewrite the above condition as

$$
C_{L}>\frac{1}{2 \omega_{p, t}^{2} L_{s}\left(1+\frac{1}{\alpha_{g d 1}}\right)} .
$$

For example, $C_{L}>160 \mathrm{fF}$ is calculated from $f_{p, t}=5 \mathrm{GHz}$, $L_{s}=0.6 \mathrm{nH}$, and $\alpha_{g d 1}=0.2$. This capacitance value can be satisfied with the parasitic capacitances of $L_{L}$ and the input capacitance of the following stage.

\section{Design}

The input transistor $M_{1}$ is designed to achieve a minimum $\mathrm{NF}$ at $5 \mathrm{GHz}$ with a bias current $I_{d 1}$ of $1.0 \mathrm{~mA}$ at a supply voltage of $0.5 \mathrm{~V}$. Equations (22) and (23) provide an optimum (for noise performance) gate width for $M_{1}$ of $4 \times$ $40 \mu \mathrm{m}$ (40 gate fingers, each with a unit of $4 \mu \mathrm{m}$ width) and a minimum gate length of $100 \mathrm{~nm}$. Although the calculated minimum NF is $3.6 \mathrm{~dB}$ for $I_{d 1}=1.0 \mathrm{~mA}$, increasing $I_{d 1}$ leads to a lower NF (i.e., $2.2 \mathrm{~dB}$ for $I_{d 1}=2.0 \mathrm{~mA}$ ).

The size of the common-gate transistor $M_{2}$ is selected as a compromise between noise and linearity performance. For a fixed bias current of $1.0 \mathrm{~mA}$, a small gate width of $M_{2}$ provides high linearity [13], but leads to a lower $g_{m 2}$, which results in the increase of $F_{M_{2}}, F_{L_{I}}$, and $F_{L_{L}}$ as shown in Eqs. (24)-(26). Figure 9 shows less NF degradation for $g_{m 2}>15 \mathrm{mS}$ than for $g_{m 2}<15 \mathrm{mS}$ at a low $k$. Thus, $g_{m 2}$ is selected to be approximately $15 \mathrm{mS}$, which results in a gate width of $4 \times 40 \mu \mathrm{m}$ and gate length of $100 \mathrm{~nm}$.

A partially-coupled transformer, shown in Fig. 10(a), allows us to simultaneously achieve a small chip area $\left(0.314 \times 0.200 \mathrm{~mm}^{2}\right)$ and reduce the magnetic coupling $(k \simeq$ 
0.1). On the other hand, a stacked transformer, shown in Fig. 10(b), provides a smaller chip area $\left(0.210 \times 0.200 \mathrm{~mm}^{2}\right)$, thereby reducing the cost. However, a large $k$ of the stacked transformer $(k \simeq 0.9)$ leads to poor gain, and does not significantly reduce the LNA NF as shown in Sect. 3. Figure 11 shows the ADS simulated voltage gain and NFs of the LNAs employing the transformers shown in Fig. 10(a) and (b). The transformers were designed using a 3-D electromagnetic simulator (Ansoft HFSS), and $L_{s}$ and $L_{g}$ in both the LNAs were adjusted to achieve an $S_{11}$ of less than $-10 \mathrm{~dB}$ at $5 \mathrm{GHz}$. The LNA with the stacked transformer had $13 \mathrm{~dB}$ lower gain than the LNA with the partially-coupled transformer at $5 \mathrm{GHz}$. This leads to an increase in the overall $\mathrm{NF}$ of the receiver. A larger $k$ also leads to an increase in the voltage swing at node I (Fig. 1), causing poor reverse isolation. Simulations (not shown) showed degradation of approximately $10 \mathrm{~dB}$ in the reverse isolation performance $\left(S_{12}\right)$ of the LNA with the stacked transformer.

The inductors of the transformer are designed to res-

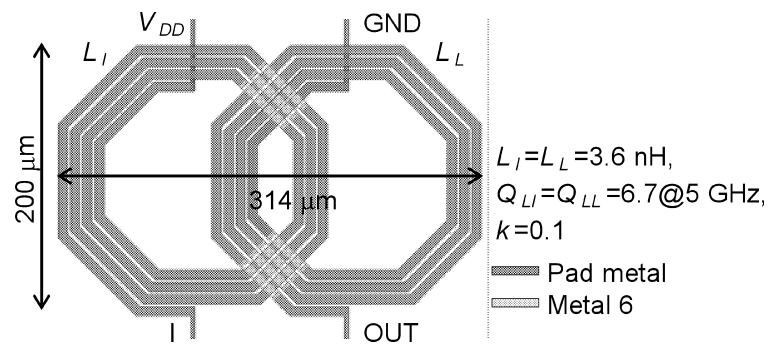

(a)

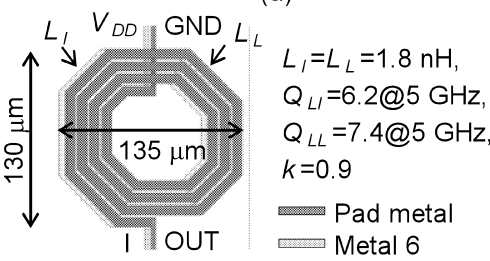

(b)

Fig. 10 Layout of (a) a partially-coupled transformer and (b) a stacked transformer

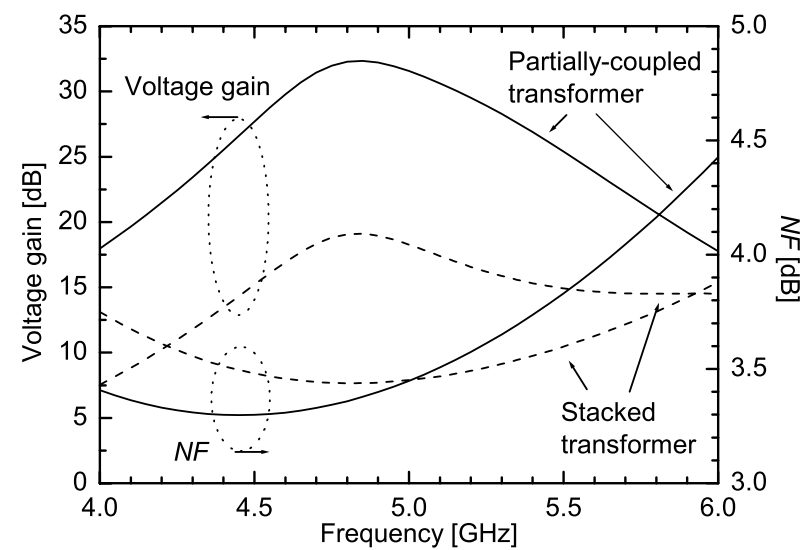

Fig. 11 Simulated voltage gain and NFs of LNAs employing the partially-coupled transformer (solid line) and stacked transformer (dashed line). onate at a frequency of approximately $5 \mathrm{GHz}$. The outer diameter of each inductor is $200 \mu \mathrm{m}$, the metal width $7 \mu \mathrm{m}$, and the metal spacing $2 \mu \mathrm{m}$. Electromagnetic simulations resulted in $L_{I}=L_{L}=3.6 \mathrm{nH}$ and quality factors $(Q)$ of 6.7 at $5 \mathrm{GHz}$.

The inductances of $L_{s}$ and $L_{g}$ are determined by the input impedance matching conditions, derived from Eqs. (7)(9): $L_{s} \simeq 0.8 \mathrm{nH}$ and $L_{g} \simeq 4.3 \mathrm{nH}$. The outer diameter of $L_{g}$ is $200 \mu \mathrm{m}$, the metal width $5 \mu \mathrm{m}$, and metal spacing $2 \mu \mathrm{m}$. The simulated $Q$ of $L_{g}$ was 7.5 at $5 \mathrm{GHz}$.

\section{Experimental Results}

The designed LNA with the partially-coupled transformer was fabricated in a $90 \mathrm{~nm}$ digital CMOS process without metal-insulator-metal (MIM) capacitors. For comparison, a conventional folded-cascode LNA was also fabricated on the same chip. A micrograph of the fabricated LNAs is shown in Fig. 12. The active chip areas (without the pads) of the proposed and conventional LNAs were $0.39 \times 0.55 \mathrm{~mm}^{2}$ and $0.52 \times 0.55 \mathrm{~mm}^{2}$, respectively. The input and output pads were not electrostatic-discharge (ESD) protected. For the measurements, a unity-gain common-source amplifier with a $50 \Omega$ output resistor was used as a buffer. The $\mathrm{S}$ -

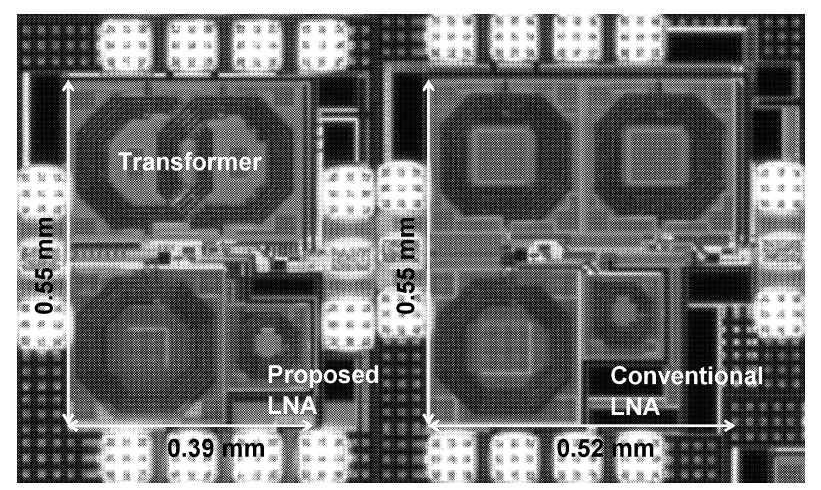

Fig. 12 Micrograph of the proposed LNA (left) and conventional folded-cascode LNA (right).

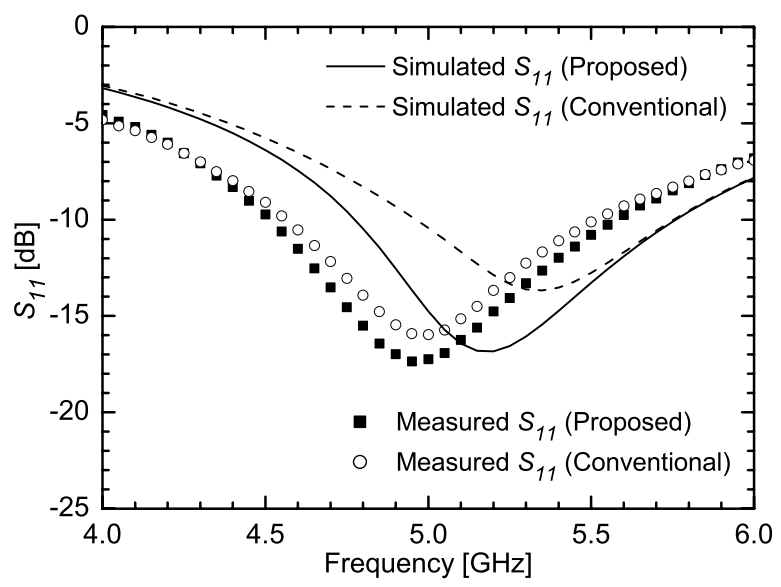

Fig. 13 Measured and simulated $S_{11}$ of the LNAs. 


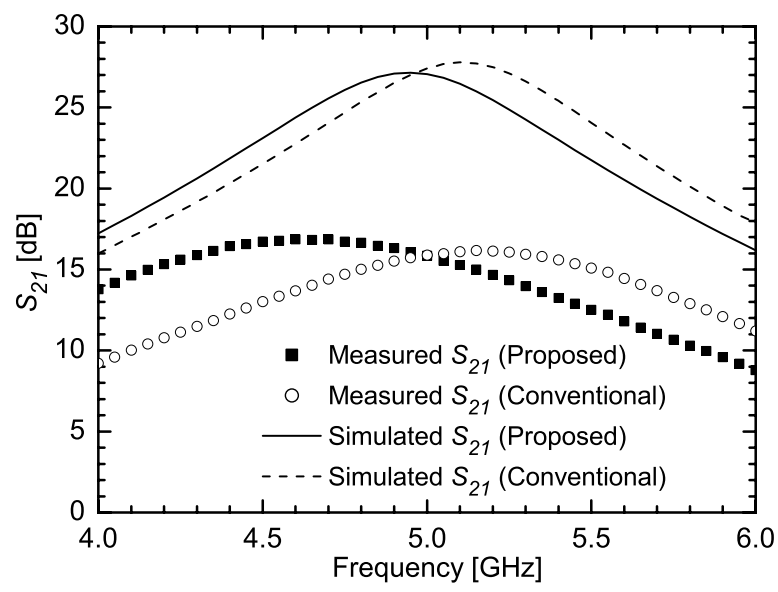

Fig. 14 Measured and simulated $S_{21}$ of the LNAs.

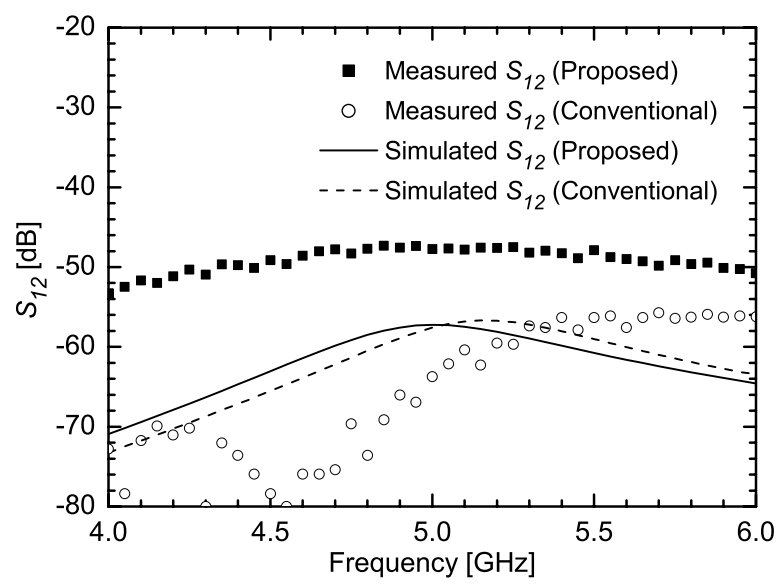

Fig. 15 Measured and simulated $S_{12}$ of the LNAs.

parameters, NFs, and linearity of the LNAs were measured using on-wafer RF probes. The power consumption of each LNA and the buffer were $1.0 \mathrm{~mW}$ and $1.8 \mathrm{~mW}$ at a supply voltage of $0.5 \mathrm{~V}$, respectively.

Figures 13 and 14 show the measured and simulated $S_{11}$ and $S_{21}$ of the LNAs, respectively. The proposed LNA obtained an $S_{11}$ of $-14 \mathrm{~dB}$ and a maximum $S_{21}$ of $16.8 \mathrm{~dB}$ at 4.7 GHz. The magnetic coupling in the proposed LNA had a small impact on the $S_{11}$ performance, while the measured peak of $S_{21}$ was shifted to a lower frequency than the simulated one, due to the increased magnetic coupling of the fabricated transformer $(k \simeq 0.2)$. This frequency shift can be reduced by using a smaller $L_{I}$ and $L_{L}(3.4 \mathrm{nH})$.

The discrepancy between the measured and simulated $S_{21}$ is mainly attributed to insufficient accuracy in the simulation of the inductors used. The electromagnetic simulator has difficulty in simulating substrate losses in conductive silicon at high frequencies, and the simulated inductors include no metal fills in order to solve convergence problems. These factors led to the decrease in the quality factors of the inductors, resulting in a lower voltage gain.

Figure 15 shows the measured and simulated $S_{12}$ of the LNAs with the buffers. The stand-alone buffer achieved an

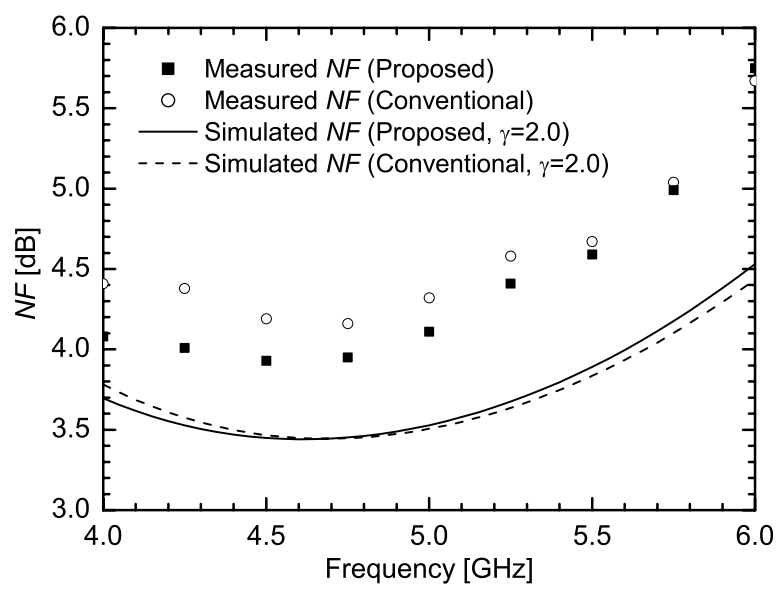

Fig. 16 Measured and simulated NFs of the LNAs.

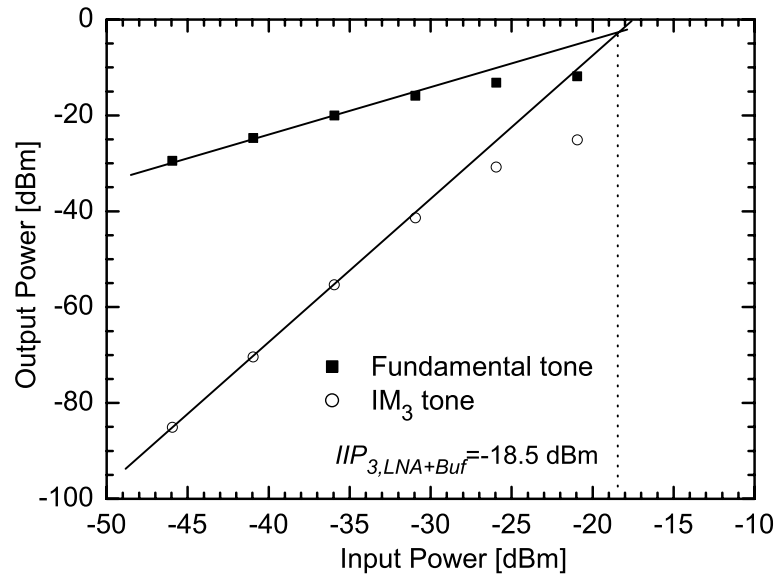

Fig. 17 Measured IIP $_{3}$ of the proposed LNA with the buffer

$S_{12}$ of $-30 \mathrm{~dB}$ around $5.0 \mathrm{GHz}$ (not shown). Thus, the $S_{12}$ of the proposed LNA without the buffer was approximately $-17 \mathrm{~dB}$. Figure 15 also shows that the inductor coupling deteriorates the reverse isolation by a factor of $12 \mathrm{~dB}$ at $5 \mathrm{GHz}$, compared to the conventional LNA. This deterioration is not problematic, because the proposed LNA still has good isolation, due to the folded-cascode topology. Measurements (not shown) also showed that both the LNAs achieved an $S_{22}$ of less than $-10 \mathrm{~dB}$ around $5.0 \mathrm{GHz}$.

Figure 16 shows the measured and simulated NFs of the LNAs. The proposed LNA obtained a minimum NF of $3.9 \mathrm{~dB}$ at $4.7 \mathrm{GHz}$, while the conventional LNA achieved a minimum NF of $4.1 \mathrm{~dB}$ at $4.7 \mathrm{GHz}$. The difference between the measured minimum NFs can be attributed to more inputreferred noise of the buffer in the conventional LNA than that in the proposed LNA. The LNAs had different values of $S_{21}$ at $4.7 \mathrm{GHz}$, which resulted in different input-referred noise of the buffer.

Figure 17 shows the measured output power of the fundamental tone and third-order intermodulation $\left(\mathrm{IM}_{3}\right)$ products for two tones $(4.999 \mathrm{GHz}$ and $5.000 \mathrm{GHz})$. The measured $\mathrm{IIP}_{3}$ of the proposed LNA with the buffer was $-18.5 \mathrm{dBm}$, and that of the stand-alone buffer was 
Table 1 Measured performance and comparison of low-voltage CMOS LNAs.

\begin{tabular}{c||c|c|c|c|c|c|c|c|c|c}
\hline \hline Reference & $\begin{array}{c}\text { CMOS } \\
\text { Technology }\end{array}$ & $\begin{array}{c}\text { Frequency } \\
{[\mathrm{GHz}]}\end{array}$ & $\begin{array}{c}\mathrm{NF} \\
{[\mathrm{dB}]}\end{array}$ & $\begin{array}{c}\text { Gain } \\
{[\mathrm{dB}]}\end{array}$ & $\begin{array}{c}\mathrm{IIP} \text { 3 } \\
{[\mathrm{dBm}]}\end{array}$ & $\begin{array}{c}\text { Pin-1 dB }_{[\mathrm{dBm}]} \\
{[\mathrm{dBm}}\end{array}$ & $\begin{array}{c}\text { Supply } \\
{[\mathrm{V}]}\end{array}$ & $\begin{array}{c}\text { Power } \\
{[\mathrm{mW}]}\end{array}$ & $\begin{array}{c}\text { Area } \\
{\left[\mathrm{mm}^{2}\right]}\end{array}$ & $\begin{array}{c}\text { FoM } \\
{\left[\mathrm{mW}^{-1}\right]}\end{array}$ \\
\hline This work & $90 \mathrm{~nm}$ & 4.7 & 3.9 & 16.8 & -14.8 & -27 & 0.5 & 1.0 & 0.21 & 4.8 \\
\hline Folded-cascode LNA & $90 \mathrm{~nm}$ & 5.0 & 4.1 & 16.1 & -14.8 & -27 & 0.5 & 1.0 & 0.29 & 4.1 \\
\hline$[4]$ & $90 \mathrm{~nm}$ & 5.5 & 3.6 & 9.2 & -7.25 & -15.8 & 0.6 & 1.0 & 0.30 & 2.2 \\
\hline$[5]$ & $180 \mathrm{~nm}$ & 5.8 & 2.9 & 7.0 & N/A & -9 & 0.7 & 12.5 & 0.40 & 0.2 \\
\hline$[6]$ & $130 \mathrm{~nm}$ & 5.1 & 5.3 & 10.3 & N/A & -22 & 0.4 & 1.0 & 0.75 & 1.4 \\
\hline$[7]$ & $180 \mathrm{~nm}$ & 5.0 & 4.5 & 9.2 & -16 & -27 & 0.6 & 0.9 & 0.54 & 1.8 \\
\hline$[14]$ & $180 \mathrm{~nm}$ & 5.0 & 3.65 & 14.1 & -17.1 & -25 & 0.6 & 1.68 & 0.46 & 2.3 \\
\hline \hline
\end{tabular}

$-0.25 \mathrm{dBm}$. Following the de-embedding procedure shown in [13], we can calculate the $\mathrm{IIP}_{3}$ of the LNA without the buffer as $-14.8 \mathrm{dBm}$. The $\mathrm{IIP}_{3}$ of the conventional LNA was also $-14.8 \mathrm{dBm}$.

Table 1 shows a summary of the LNA performance and a comparison with previously reported low-voltage (approximately $0.6 \mathrm{~V}$ ) CMOS LNAs for $5 \mathrm{GHz}$ applications. The proposed LNA achieved performance comparable to the conventional folded-cascode LNA, while consuming three fourths of the chip area of the conventional LNA. The figure of merit (FoM) for the LNAs, included in Table 1, is defined as [4]

$$
\text { FoM }\left[\mathrm{mW}^{-1}\right]=\frac{\operatorname{Gain}[\mathrm{lin}]}{\text { Power }[\mathrm{mW}] \cdot(N F[\operatorname{lin}]-1)} .
$$

Among the reported low-voltage CMOS LNAs, the proposed LNA obtained the best FoM $\left(4.8 \mathrm{~mW}^{-1}\right)$ with the smallest chip area

\section{Conclusion}

We have demonstrated a transformer folded-cascode CMOS LNA, in which the internal and load inductors have been magnetically coupled to reduce the chip area. Circuit analysis showed that the magnetic coupling between these inductors decreases the resonance frequency of the input matching network, the peak frequency and magnitude of the gain, and the noise figure. The partially-coupled transformer reduced the chip area, while having a small impact on the LNA performance. The LNA, implemented with a $90 \mathrm{~nm}$ CMOS technology, occupied $0.21 \mathrm{~mm}^{2}$, and achieved an $S_{11}$ of $-14 \mathrm{~dB}$, NF of $3.9 \mathrm{~dB}$, and voltage gain of $16.8 \mathrm{~dB}$ at $4.7 \mathrm{GHz}$ with a power consumption of $1.0 \mathrm{~mW}$ from a $0.5 \mathrm{~V}$ supply. It has been demonstrated that the proposed LNA can replace conventional low-voltage CMOS LNAs.

\section{Acknowledgments}

The chip in this study was fabricated through the chip fabrication program of the VLSI Design and Education Center (VDEC), the University of Tokyo, in collaboration with Semiconductor Technology Academic Research Center (STARC), Fujitsu Limited, Matsushita Electric Industrial Company Limited., NEC Electronics Corporation, Renesas Technology Corporation, and Toshiba Corporation. In addition, this study was financially supported by STARC and by a grant to the Osaka University Global COE Program, "Center for Electronic Devices Innovation," from the Ministry of Education, Culture, Sports, Science and Technology of Japan.

\section{References}

[1] Semiconductor Industry Association, "International technology roadmap for semiconductors 2007 edition." Available: http://www. itrs.net/

[2] H. Tsuchikawa, M. Takakuwa, and S. Sugatani, "Electron beam direct writing technology combined with silicon shuttle service," 4th ISMI Symp. on Manufacturing Effectiveness, Austin, TX, Oct. 2007.

[3] D.K. Shaeffer and T.H. Lee, "A 1.5 V, 1.5 GHz CMOS low noise amplifier," IEEE J. Solid-State Circuits, vol.32, no.5, pp.745-759, May 1997.

[4] D. Linten, L. Aspemyr, W. Jeamsaksiri, J. Ramos, A. Mercha, S. Jenei, S. Thijs, R. Garcia, H. Jacobsson, P. Wambacq, S. Donnay, and S. Decoutere, "Low-power $5 \mathrm{GHz}$ LNA and VCO in $90 \mathrm{~nm}$ RF CMOS," Symp. VLSI Circuits Dig. Tech. Papers, pp.372-375, Honolulu, HI, June 2004

[5] T.K.K. Tsang and M.N. El-Gamal, "Gain and frequency controllable sub-1 V 5.8 GHz CMOS LNA," Proc. IEEE Int. Symp. Circuits and Systems, Scottsdale, AZ, pp.795-798, May 2002.

[6] D. Wu, R. Huang, W. Wong, and Y. Wang, "A 0.4-V low noise amplifier using forward body bias technology for $5 \mathrm{GHz}$ application," IEEE Microw. Wireless Compon. Lett., vol.17, no.7, pp.543-545, July 2007.

[7] H.H. Hsieh and L.H. Lu, "A CMOS 5-GHz micro-power LNA," IEEE Radio Frequency Integrated Circuits Symp. Dig. Papers, Long Beach, CA, pp.31-34, June 2005.

[8] T. Kihara, H.J. Park, I. Takobe, F. Yamashita, T. Matsuoka, and $\mathrm{K}$. Taniguchi, "A $0.5 \mathrm{~V}$ area-efficient transformer folded-cascode low-noise amplifier in $90 \mathrm{~nm}$ CMOS," Proc. IEEE Int. Conf. on Integrated Circuit Design and Technology, Grenoble, France, pp.21-24, June 2008.

[9] G. Gonzalez, Microwave Transistor Amplifiers, 2nd ed., Prentice Hall, Upper Saddle River, 1997.

[10] T. Kihara, T. Matsuoka, and K. Taniguchi, "A 1.0 V, $2.5 \mathrm{~mW}$, transformer noise-canceling UWB CMOS LNA," IEEE Radio Frequency Integrated Circuits Symp. Dig. Papers, Atlanta, GA, pp.493-496, June 2008.

[11] A. van der Ziel, Noise in Solid State Devices and Circuits, John Wiley \& Sons, Toronto, 1986.

[12] Y. Tsividis, Operation and Modeling of the MOS Transistor, 2 ed., Oxford University Press, New York, 2003.

[13] T. Kihara, G. Kim, M. Goto, K. Nakamura, Y. Shimizu, T. Matsuoka, and K. Taniguchi, "Analytical expression based design of a lowvoltage FD-SOI CMOS low-noise amplifier," IEICE Trans. Fundamentals, vol.E90-A, no.2, pp.317-325, Feb. 2007.

[14] H.H. Hsieh, J.H. Wang, and L.H. Lu, "Gain-enhancement techniques for CMOS folded cascode LNAs at low-voltage operations," 
IEEE Trans. Microw. Theory Tech., vol.56, no.8, pp.1807-1816, Aug. 2008.

\section{Appendix A: Frequency Responses of $Y_{I}$ and 1/ $Y_{I}$}

\section{A.1 $Y_{I}$}

The frequency response of $Y_{I}$ is shown in Fig. A.1(a). At low frequencies, Eq. (3) can be approximated as

$$
\begin{aligned}
& \operatorname{Re}\left[Y_{I}\right] \approx g_{m 2}(1-n k), \\
& \operatorname{Im}\left[Y_{I}\right] \approx \omega\left(C_{I}+n^{2} k^{2} C_{L}\right)-\frac{1}{\omega L_{I}},
\end{aligned}
$$

which shows that $\operatorname{Im}\left[Y_{I}\right]$ becomes zero around

$$
w_{0, Y_{I}}=\frac{1}{\sqrt{L_{I}\left(C_{I}+n^{2} k^{2} C_{L}\right)}} .
$$

At $\omega_{1, Y_{I}}=1 / \sqrt{\left(1-k^{2}\right) L_{L} C_{L}}, \operatorname{Re}\left[Y_{I}\right]$ exceeds $g_{m 2}$ and $\operatorname{Im}\left[Y_{I}\right]$ becomes a maximum:

$$
\begin{aligned}
& \operatorname{Re}\left[Y_{I}\right] \approx g_{m 2}+\frac{n^{2} k^{2}}{R_{L}}, \\
& \operatorname{Im}\left[Y_{I}\right] \approx \omega_{1, Y_{I}} C_{I}-\frac{1}{\omega_{1, Y_{I}} L_{I}}+\frac{n k g_{m 2}}{\omega_{1, Y_{I}} R_{L} C_{L}},
\end{aligned}
$$

respectively. Above $\omega_{1, Y_{I}}, \operatorname{Re}\left[Y_{I}\right]$ and $\operatorname{Im}\left[Y_{I}\right]$ approach gradually $g_{m 2}$ and $\omega C_{I}-1 / \omega L_{I}$, respectively.
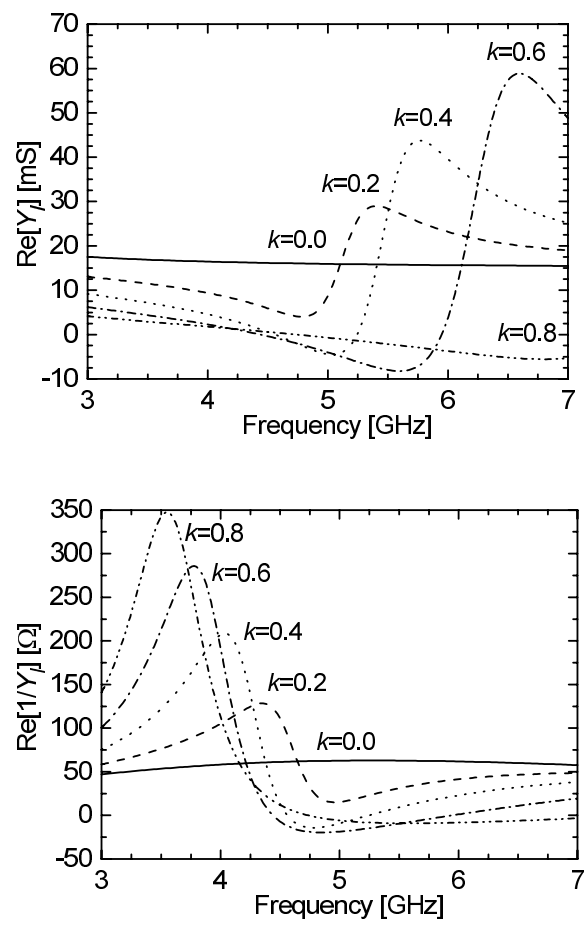

$g_{m 1}=20 \mathrm{mS}, L_{g}=4.0 \mathrm{nH}, L_{s}=0.6 \mathrm{nH}, C_{g s 1}=150 \mathrm{fF}, C_{g d 1}=35 \mathrm{fF}, g_{m 2}=15 \mathrm{mS}, C_{F}=C_{L}=250 \mathrm{fF}, L_{l}=L_{L}=4.0 \mathrm{nH}, R_{F}=R_{L}=15 \Omega$

\section{A. $21 / Y_{I}$}

The reverse of $Y_{I}$ is expressed as

$$
\frac{1}{Y_{I}}=\frac{s C_{L}+\frac{1}{s\left(1-k^{2}\right) L_{L}+R_{L}}}{D}
$$

where $D$ is given by Eq. (16). Figure A. 1(b) shows the calculated frequency response of $1 / Y_{I}$ with $k$ as a parameter. At low frequencies, $1 / Y_{I}$ is approximated as

$$
\begin{aligned}
& \operatorname{Re}\left[\frac{1}{Y_{I}}\right] \approx \frac{R_{I}}{1+R_{I} g_{m 2}(1-n k)}, \\
& \operatorname{Im}\left[\frac{1}{Y_{I}}\right] \approx 0 .
\end{aligned}
$$

Equation (16) indicates that $\operatorname{Re}\left[1 / Y_{I}\right]$ and $\operatorname{Im}\left[1 / Y_{I}\right]$ have peaks around $\omega_{p}$, given by Eq. (19). Above $\omega_{p}, \operatorname{Re}\left[1 / Y_{I}\right]$ and $\operatorname{Im}\left[1 / Y_{I}\right]$ approach gradually $1 / g_{m 2}$ and $1 /\left(\omega C_{I}-\right.$ $\left.1 / \omega L_{I}\right)$, respectively.

\section{Appendix B： NF Derivation}

The noise of $M_{1}, M_{2}, R_{I}$, and $R_{L}$ contribute to the overall LNA noise. The LNA noise factor is given by

$$
\begin{aligned}
F & =\frac{\overline{\left|v_{o, R_{s}}\right|^{2}}+\overline{\left|v_{o, M_{1}}\right|^{2}}+\overline{\left|v_{o, M_{2}}\right|^{2}}+\overline{\left|v_{o, R_{1}}\right|^{2}}+\overline{\left|v_{o, R_{L}}\right|^{2}}}{\overline{\left|v_{o, R_{s}}\right|^{2}}}, \\
& =1+F_{M_{1}}+F_{M_{2}}+F_{L_{I}}+F_{L_{L}},
\end{aligned}
$$

(a)
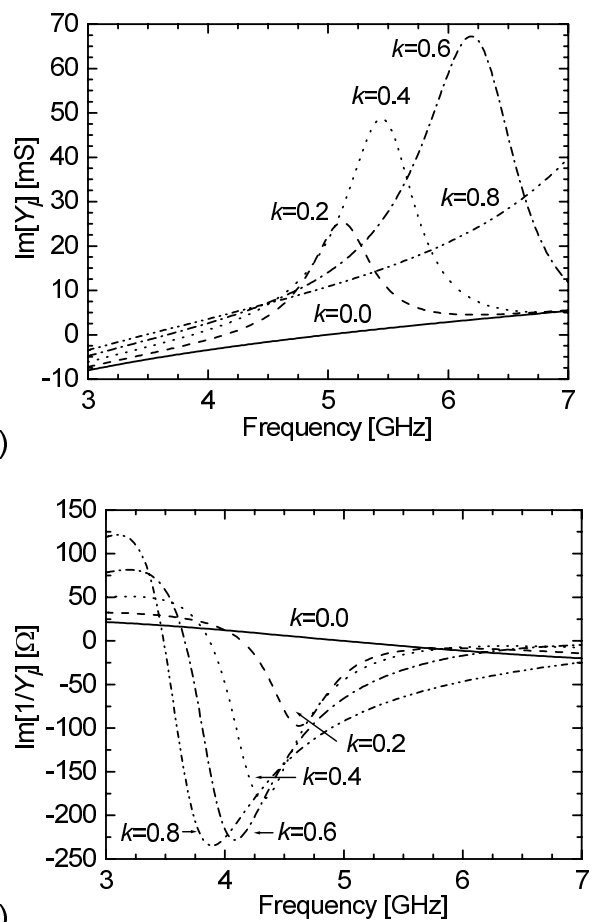

(b)

Fig. A. 1 Calculated (a) $Y_{I}$ and (b) $1 / Y_{I}$ with $k$ as a parameter. 


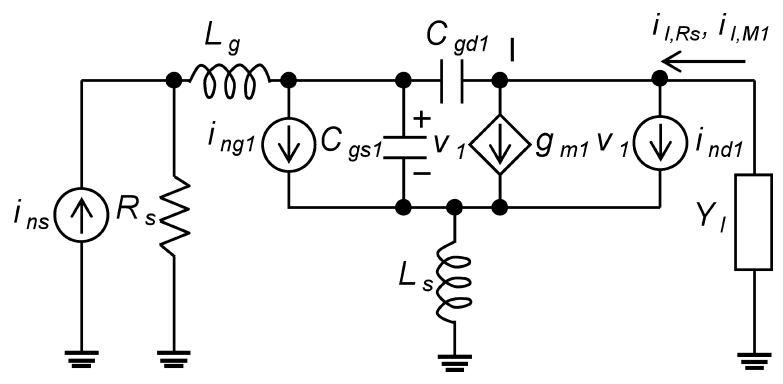

Fig. A. 2 Noise equivalent circuit of the input stage.

where $v_{o, R_{s}}, v_{o, M_{1}}, v_{o, M_{2}}, v_{o, R_{I}}$, and $v_{o, R_{L}}$ are the output noise voltages originating from $R_{s}, M_{1}, M_{2}, R_{I}$, and $R_{L}$, respectively.

The output noise voltage originating from $R_{s}, v_{o, R_{s}}$, can be derived from the noise equivalent circuit of the input stage, shown in Fig. A.2. The signal source noise current is expressed as

$$
\overline{\left|i_{n s}\right|^{2}}=\frac{4 k_{B} T \Delta f}{R_{s}},
$$

where $k_{B}$ is Boltzmann's constant, $T$ the absolute temperature, and $\Delta f$ the noise bandwidth. The transfer function from $i_{n s}$ to the noise current at node $\mathrm{I}, i_{I, R s}$, is given by

$$
H_{n s}\left(j \omega_{0}\right)=\frac{g_{m 1} R_{s}}{j \omega_{0} \alpha_{M} C_{g s 1}\left(R_{s}+\omega_{T} L_{s} / \alpha_{M}\right)},
$$

where $\operatorname{Im}\left[Z_{\text {in }}\left(j \omega_{0}\right)\right]=0$ and $\alpha_{M}$ is approximated as $\operatorname{Re}\left[\alpha_{M}\right]$ for input impedance matching as shown in Section 3.1. Using Eq. (A. 11), we have

$$
\begin{aligned}
\overline{\left|i_{I, R S}\right|^{2}} & =\left|H_{n s}\left(j \omega_{0}\right)\right|^{2} \overline{\left|i_{n s}\right|^{2}} \\
& =\frac{4 k_{B} T R_{s} \omega_{T 1}^{2} \Delta f}{\omega_{0}^{2}\left|\alpha_{M}\right|^{2}\left(R_{s}+\omega_{T} L_{s} /\left|\alpha_{M}\right|\right)^{2}} .
\end{aligned}
$$

The common-gate stage converts $i_{I, R s}$ to the output voltage:

$$
\overline{\left|v_{o, R s}\right|^{2}}=\left|Z_{T}\right|^{2} \overline{\left|i_{I, R s}\right|^{2}}
$$

where $Z_{T}$ is the transimpedance of the common-gate stage and is given by Eq. (15).

\section{B.1 $F_{M_{1}}$}

The noise current of $M_{1}$ is also converted by the commongate stage. The noise contribution from $M_{1}$ can be expressed as

$$
F_{M_{1}}=\frac{\overline{\left|v_{o, M_{1}}\right|^{2}}}{\overline{\left|v_{o, R_{s}}\right|^{2}}}=\frac{\left|Z_{T}\right|^{2} \overline{\left|i_{I, M_{1}}\right|^{2}}}{\left|Z_{T}\right|^{2} \overline{\left|i_{I, R_{s}}\right|^{2}}}=\frac{\overline{\left|i_{I, M_{1}}\right|^{2}}}{\overline{\left|i_{I, R_{s}}\right|^{2}}},
$$

where $i_{I, M_{1}}$ is the noise current at node $\mathrm{I}$ as shown in Fig. A. 2.

The main noise sources in a MOSFET are the drain noise current $i_{n d}$ and induced-gate noise current $i_{n g}$, which are expressed as

$$
\begin{aligned}
& \overline{\left|i_{n d}\right|^{2}}=4 k_{B} T \gamma g_{d 0} \Delta f, \\
& \overline{\left|i_{n g}\right|^{2}}=4 k_{B} T \delta \frac{\left(\omega C_{g s}\right)^{2}}{\kappa g_{d 0}} \Delta f,
\end{aligned}
$$

respectively. The induced-gate noise current correlates to the drain noise current, and the correlation coefficient is given by [11]

$$
c=\frac{\overline{i_{n g} \cdot i_{n d}^{*}}}{\sqrt{\overline{\left|i_{n g}\right|^{2}}} \sqrt{\overline{\left|i_{n d}\right|^{2}}}} .
$$

Using this coefficient, we can express the induced-gate noise as

$$
\begin{aligned}
\overline{\left|i_{n g}\right|^{2}} & =\overline{\left|i_{n g c}\right|^{2}}+\overline{\left|i_{n g u}\right|^{2}} \\
& =\overline{\left|i_{n g}\right|^{2}}|c|^{2}+\overline{\left|i_{n g}\right|^{2}}\left(1-|c|^{2}\right),
\end{aligned}
$$

where $i_{n g c}$ and $i_{n g u}$ are the correlated and uncorrelated components, respectively. The noise current due to $M_{1}$ at node I is therefore expressed as

$$
\begin{aligned}
\overline{\left|i_{I, M_{1}}\right|^{2}}= & \overline{\left|i_{I, n d 1}+i_{I, n g 1}\right|^{2}} \\
= & \overline{\left|i_{I, n d 1}+i_{I, n g c 1}\right|^{2}}+\overline{\left|i_{I, n g u 1}\right|^{2}} \\
= & \overline{\left|i_{I, n d 1}\right|^{2}}+\overline{i_{I, n g c 1} \cdot i_{I, n d 1}^{*}}+\overline{i_{I, n d 1} \cdot i_{I, n g c 1}^{*}} \\
& +\overline{\left|i_{I, n g c 1}\right|^{2}}+\overline{\left|i_{I, n g u 1}\right|^{2}},
\end{aligned}
$$

where $i_{I, n d 1}, i_{I, n g c 1}$, and $i_{I, n g u 1}$ are the noise currents originating from $i_{n d 1}, i_{n g c 1}$, and $i_{n g u 1}$ at node I, respectively. From Fig. A. 2, the transfer function from $i_{n d 1}$ to $i_{I, n d 1}$ is approximated as

$$
H_{n d 1}\left(j \omega_{0}\right) \approx \frac{R_{s}\left(1+\alpha_{g d 1}\right)}{\alpha_{M}\left(R_{s}+\omega_{T 1} L_{s} / \alpha_{M}\right)} .
$$

The transfer function from $i_{n g 1}$ to $i_{I, n g 1}$ is also approximated as

$$
H_{n g 1}\left(j \omega_{0}\right) \approx-\frac{g_{m 1}\left(R_{s}+\frac{j}{\omega_{0} C_{g s 1}}\right)}{j \omega_{0} \alpha_{M} C_{g s 1}\left(R_{s}+\omega_{T 1} L_{s} / \alpha_{M}\right)} .
$$

Using Eqs. (A· 15), (A· 16), (A· 20), and (A·21), we have

$$
\begin{aligned}
& \overline{\left|i_{I, n d 1}\right|^{2}}=\left|H_{n d 1}\left(j \omega_{0}\right)\right|^{2} \overline{\left|i_{n d 1}\right|^{2}} \\
& =\frac{4 k_{B} T \gamma_{1} g_{m 1} R_{s}^{2}\left(1+\alpha_{g d 1}\right)^{2} \Delta f}{\alpha_{1}\left|\alpha_{M}\right|^{2}\left(R_{s}+\omega_{T 1} L_{s} /\left|\alpha_{M}\right|\right)^{2}}, \\
& \overline{i_{I, n g c 1} \cdot i_{I, n d 1}^{*}}+\frac{i_{I, n d 1} \cdot i_{I, n g c 1}^{*}}{=H_{n g c 1}\left(j \omega_{0}\right) i_{n g c 1} \cdot H_{n d 1}^{*}\left(j \omega_{0}\right) i_{n d 1}^{*}} \\
& +H_{n d 1}\left(j \omega_{0}\right) i_{n d 1} \cdot H_{n g c 1}^{*}\left(j \omega_{0}\right) i_{n g c 1}^{*} \\
& =-2|c| \\
& \sqrt{\frac{\delta_{1}}{\kappa_{1} \gamma_{1}}} \frac{4 k_{B} T \gamma_{1} g_{m 1} R_{s}^{2}\left(1+\alpha_{g d 1}\right) \Delta f}{\left|\alpha_{M}\right|^{2}\left(R_{s}+\omega_{T 1} L_{s} /\left|\alpha_{M}\right|\right)^{2}}, \\
& \overline{\left|i_{I, n g c 1}\right|^{2}}+\overline{\left|i_{I, n g u 1}\right|^{2}}
\end{aligned}
$$




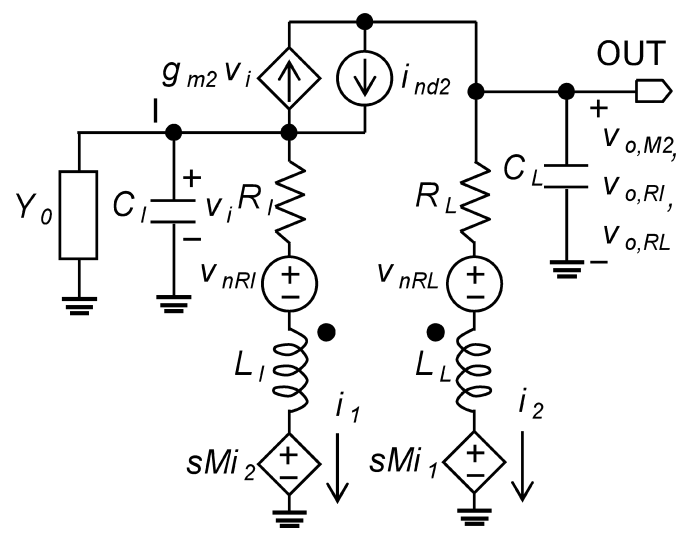

Fig. A. 3 Noise equivalent circuit of the common-gate stage.

$$
\begin{aligned}
& =\left|H_{n g 1}\left(j \omega_{0}\right)\right|^{2} \overline{\left|i_{n g 1}\right|^{2}} \\
& =\frac{4 k_{B} T \alpha_{1} \delta_{1} g_{m 1}\left(R_{s}^{2}+\frac{1}{\omega_{0}^{2} C_{g s 1}^{2}}\right) \Delta f}{\kappa_{1}\left|\alpha_{M}\right|^{2}\left(R_{s}+\omega_{T 1} L_{s} /\left|\alpha_{M}\right|\right)^{2}} .
\end{aligned}
$$

Substituting Eqs. (A. 22)-(A. 24) into Eq. (A. 19) gives the noise current of $M_{1}$ at node I:

$$
\begin{aligned}
\overline{\left|i_{I, M_{1}}\right|^{2}}= & \frac{4 k_{B} T R_{s} \Delta f}{\left|\alpha_{M}\right|^{2}\left(R_{s}+\omega_{T 1} L_{s} /\left|\alpha_{M}\right|\right)^{2}} \\
& \times\left(\frac{\gamma_{1} \chi_{1}}{\alpha_{1}} g_{m 1} R_{s}+\frac{\alpha_{1} \delta_{1} g_{m 1}}{\kappa_{1} \omega_{0}^{2} C_{g s 1}^{2} R_{s}}\right),
\end{aligned}
$$

where $\chi_{1}$ is given by Eq. (23). Substituting Eqs. (A.12) and (A. 25) into Eq. (A. 14) gives $F_{M_{1}}$ (Eq. (22)).

\section{B.2 $F_{M_{2}}$}

In a common-gate topology, the gate-induced noise current of the MOSFET can be ignored against the drain noise current:

$$
\overline{\left|v_{o, M_{2}}\right|^{2}} \approx \overline{\left|v_{o, n d 2}\right|^{2}},
$$

where $v_{o, n d 2}$ is the output voltage originating from the drain noise current of $M_{2}, i_{n d 2}$, and is derived from the noise equivalent circuit of the common-gate stage, shown in Fig. A. 3:

$$
\begin{aligned}
\overline{\left|v_{o, n d 2}\right|^{2}}= & \left|\frac{N_{n d 2}}{Y_{0}+Y_{I}}\right|^{2} \overline{\left|i_{n d 2}\right|^{2}} \\
= & \left|\frac{N_{n d 2}}{Y_{0}+Y_{I}}\right|^{2} \cdot 4 k_{B} T \frac{\gamma_{2}}{\alpha_{2}} g_{m 2} \Delta f, \\
N_{n d 2}= & -\left(s L_{L}+R_{L}-\frac{s^{2} M^{2}}{s L_{I}+R_{I}}\right)\left(Y_{0}+Y_{L_{I} C_{I}}\right) \\
& +\frac{s M}{s L_{I}+R_{I}}\left(1-\frac{s M}{s L_{I}+R_{I}}\right) \\
\approx & -s^{2}\left(1-k^{2}\right) L_{I} \cdot\left(Y_{0}+Y_{L_{I} C_{I}}\right) \\
& +n k(1-n k),
\end{aligned}
$$

where $M$ is the mutual inductance between $L_{I}$ and $L_{L}, Y_{0}$ represents the output admittance of the input stage at node $\mathrm{I}$, and $Y_{L_{I} C_{I}}=s C_{I}+1 /\left(s L_{I}+R_{I}\right)$, as shown in Section 3.3. Rewriting Eq. (A.13) in terms of $Y_{0}+Y_{I}$, we have

$$
\begin{aligned}
& \overline{\left|v_{o, R s}\right|^{2}}=\left|\frac{N_{n s}}{Y_{0}+Y_{I}}\right|^{2}\left|\frac{Y_{0}+Y_{I}}{Y_{I}}\right|^{2} \overline{\left|i_{I, R s}\right|^{2}}, \\
& N_{n s}=\frac{s M}{s L_{I}+R_{I}}+g_{m 2}\left(s L_{L}+R_{L}-\frac{s^{2} M^{2}}{s L_{I}+R_{I}}\right) \\
& \approx n k+g_{m 2} \cdot s n^{2}\left(1-k^{2}\right) L_{I} .
\end{aligned}
$$

Dividing Eq. (A. 27) by Eq. (A. 29) with $L_{I}=1 / \omega_{0}^{2}(1+k) C_{L}$ and $n=1$, we obtain $F_{M_{2}}$ (Eq. (24)).

\section{B.3 $\quad F_{L_{I}}$ and $F_{L_{L}}$}

The noise voltages of the parasitic resistances of $L_{I}$ and $L_{L}$ are given by

$$
\begin{aligned}
& \overline{\left|v_{n R_{I}}\right|^{2}}=4 k_{B} T R_{I} \Delta f, \\
& \mid \overline{\left.v_{n R_{L}}\right|^{2}}=4 k_{B} T R_{L} \Delta f,
\end{aligned}
$$

respectively. The output noise voltages due to $v_{n R_{I}}$ and $v_{n R_{L}}$ can be expressed from Fig. A. 3:

$$
\begin{aligned}
\overline{\left|v_{o, R_{L}}\right|^{2}} & =\left|\frac{N_{n R_{L}}}{Y_{0}+Y_{I}}\right|^{2} \overline{\left|v_{n R_{L}}\right|^{2}}, \\
N_{n R_{L}} & =g_{m 2}\left(1-\frac{s M}{s L_{I}+R_{I}}\right)+Y_{0}+Y_{L_{I} C_{I}} \\
& \approx g_{m 2}(1-n k)+Y_{0}+Y_{L_{I} C_{I}}
\end{aligned}
$$

and

$$
\begin{aligned}
\overline{\left|v_{o, R_{I}}\right|^{2}}= & \left|\frac{N_{n R_{I}}}{Y_{0}+Y_{I}}\right|^{2} \overline{\left|v_{n R_{I}}\right|^{2}} \\
N_{n R_{I}}= & g_{m 2}\left(\frac{s L_{L}+R_{L}}{s L_{I}+R_{I}}-\frac{s M}{s L_{I}+R_{I}}\right) \\
& -\frac{s M}{s L_{I}+R_{I}}\left(Y_{0}+s C_{I}\right) \\
\approx & n(n-k) g_{m 2}-n k\left(Y_{0}+s C_{I}\right),
\end{aligned}
$$

respectively. Dividing Eqs. (A. 33) and (A.35) by Eq. (A. 29) with $L_{I}=1 / \omega_{0}^{2}(1+k) C_{L}$ and $n=1$, we derive $F_{L_{I}}$ (Eq. (25)) and $F_{L_{L}}$ (Eq. (26)), respectively.

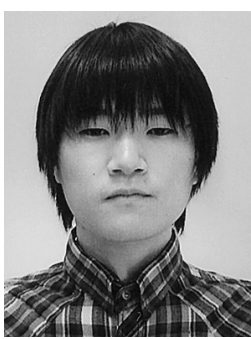

Takao Kihara received the B.S. and M.S degrees in electronic engineering from Osaka University, Osaka, Japan, in 2005 and 2006, respectively. He is now working towards his Ph.D. degree at Osaka University. His current research interests include low-voltage CMOS RF circuits. He is a student member of the IEEE. 


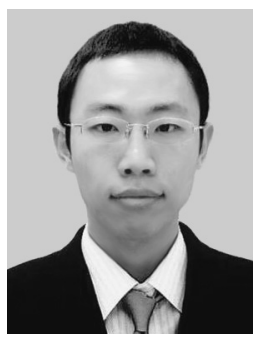

Hae-Ju Park received the B.S. degree in electronic engineering from Shizuoka University, Shizuoka, Japan, in 2007. He is now working towards his M.S. degree at Osaka University. His current research interests include CMOS RF circuits.

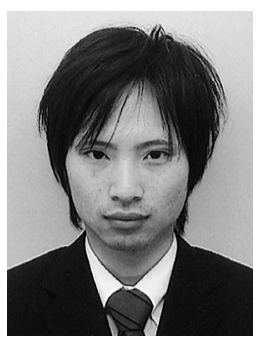

Isao Takobe received the B.S. degree in electronic engineering from Tokushima University, Tokushima, Japan, in 2007. He is now working towards his M.S. degree at Osaka University. His current research interests include CMOS RF circuits.

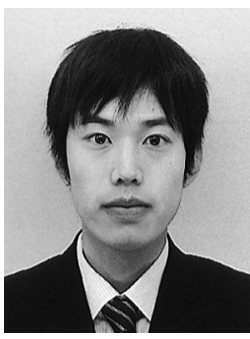

Fumiaki Yamashita received the B.S. degree in physics from Osaka City University, Osaka, Japan, in 2004. He is now working towards his M.S. degree at Osaka University. His current research interests include low-voltage CMOS VCOs.

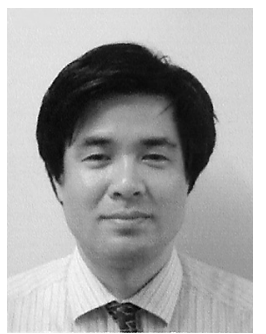

Toshimasa Matsuoka received the B.S., M.S. and Ph.D. degrees in electronic engineering from Osaka University, Osaka, Japan, in 1989, 1991 and 1996, respectively. During 1991-1998, he worked for the Central Research Laboratories, Sharp Corporation, Nara, Japan, where he was engaged in the research and development of deep submicron CMOS devices and ultra thin gate oxides. Since 1999, he has been working for Osaka University, where he is Associate Professor now. His current research includes MOS device modeling and CMOS RF circuits. Dr. Matsuoka is a member of the JSAP, the IEEJ, and the IEEE.

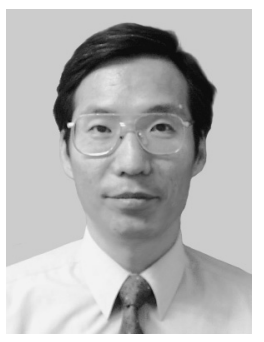

Kenji Taniguchi received the B.S., M.S. and Ph.D. degrees from Osaka University, Osaka, Japan, in 1971, 1973 and 1986, respectively. From 1973 to 1986, he worked for Toshiba Research and Development Center, Kawasaki, Japan, where he was engaged in process modeling and the design of MOS LSI fabrication technology. He was a Visiting Scientist at Massachusetts Institute of Technology, Cambridge, from 1982 to 1983. Now, he is Professor at Osaka University. His current research interests are analog circuits, device physics and process technology. Prof. Taniguchi is a member of the JSAP, the IEEJ, and the IEEE. 